\title{
Botulinum Toxins in Medical and Cosmetic Dermatology
}

\author{
Authors: \\ *Margit Juhász, ${ }^{1}$ Anna-Marie Hosking, ${ }^{1}$ Natasha Atanaskova \\ Mesinkovska ${ }^{1,2}$ \\ 1. University of California, Irvine, Department of Dermatology, Irvine, California, USA \\ 2. University of California, Irvine, Beckman Laser Institute, Irvine, California, USA \\ *Correspondence to mjuhasz@hs.uci.edu \\ Disclosure: $\quad$ The authors have declared no conflicts of interest. \\ Received: $\quad 06.08 .20$ \\ Accepted: $\quad 22.01 .21$ \\ Keywords: $\quad$ Botulinum toxin (BoNT), hyperhidrosis, Raynaud's phenomenon, rhytides, scarring. \\ Citation: $\quad$ EMJ Dermatol. 2021; DOI/10.33590/emjdermatol/20-00202
}

\section{Abstract}

Background: Botulinum toxin (BoNT), a bacterially produced neurotoxin, is a mainstay in the dermatologic armamentarium. Although BoNT is commonly used to treated rhytides associated with ageing, it can be employed for a variety of other cosmetic purposes and medical disorders.

Objective: In this review, the authors aim to describe the multitude of uses for BoNT in the dermatologic field.

Materials and Methods: This manuscript was designed as a retrospective review of the on- and offlabel applications of BoNT in dermatology.

Results: In addition to treatment of rhytides, BoNT has been shown to decrease rosacea, menopauseassociated flushing, and facial sebum production, while improving patient confidence in their appearance. Furthermore, BoNT has been successfully used to treat primary hyperhidrosis, hair loss, aberrant scarring, Raynaud's phenomenon-associated vasospasm, as well as a variety of skin diseases. Side effects of BoNT include pain or discomfort associated with injections during treatment, bruising, asymmetry, and swelling. Patients are generally satisfied with clinical results after BoNT treatment.

Conclusion: Dermatologists should be aware of all on- and off-label applications of BoNT to provide patients with timely and appropriate medical care. Further research must be completed to fully characterise the safety and use of BoNT for off-label purposes.

\section{INTRODUCTION}

Botulinum neurotoxins (BoNTs) are derived from Clostridium botulinum, a gram-positive anaerobe. While there are seven subtypes of BoNT (A to $G$ ), only $A$ and $B$ are currently clinically relevant. The cosmetic potential of BoNT-A was first described in the early 1990s by Drs Jean

and Alistair Carruthers as a safe and effective treatment for dynamic rhytides. ${ }^{1}$ Ten years later, BoNT-A was approved by the U.S. Food and Drug Administration (FDA) for the treatment of glabellar rhytides. Since their commercial availability, BoNTs have been used on- and offlabel in both the medical and cosmetic realms for spasticity, migraines, depression, hyperhidrosis, 
as well as ageing of the face, neck, and décolletage. BoNT is secreted by $C$. botulinum as a three-protein complex with the $150 \mathrm{kDa}$ toxin, a non-toxin haemagglutinin protein, and a non-toxin, non-haemagglutinin protein. Bacterial proteases cleave the toxin into an active, dichain product consisting of the 100 kDa 'heavy' and $50 \mathrm{kDa}$ 'light' chains. Once the active toxin is introduced to the presynaptic nerve terminal, the heavy chain binds synaptic vesicle glycoprotein 2 causing endocytosis of the toxin-glycoprotein complex and toxin light chain release into the synaptic space. Toxin light chains cleave either synaptosomal-associated protein 25 (BoNT-A, C, E), or vesicle-associated membrane protein/synaptobrevin (BoNT-B, D, F, $G)$, disallowing the release of acetylcholine from the axon of peripheral motor nerves, and causing subsequent temporary chemical denervation and muscle paralysis. $^{2}$
There are four commercially available, FDAapproved BoNT-A preparations in the USA: onabotulinumtoxinA (ONA; Botox ${ }^{\circledR}$, Allergan Inc., Irvine, California, USA); abobotulinumtoxinA (ABO; Dysport ${ }^{\circledast}$, Medicis Pharmaceuticals Corp., Scottsdale, Arizona, USA); incobotulinumtoxinA (INCO; Xeomin ${ }^{\oplus}$, Merz Aesthetics, Frankfurt, Germany); and, newest to the market, prabotulinumtoxinA-xvfs (PRA; Jeuveau ${ }^{\text {TM }}$, Evolus, Newport Beach, California, USA); as well as one BoNT-B: rimabotulinumtoxinB (RIMA; Myobloc $^{\circledast}$, Solstic Pharmaceuticals, San Francisco, California, USA). While ONA, ABO, and INCO are approved for use in the medical and cosmetic settings, PRA is only approved for glabellar lines and RIMA is only approved for the treatment of cervical dystonia (Table 1). For the purposes of this present review, it is important to note that all treatments discussed will refer to units (U) of BoNT-A as ONA equivalents, unless otherwise stated.

Table 1: Characteristics of commercially available botulinum toxins in the USA.

\begin{tabular}{|c|c|c|c|c|c|}
\hline $\begin{array}{l}\text { Commercially } \\
\text { available toxin }\end{array}$ & FDA approval & Serotype & Molecular target & $\begin{array}{l}\text { Molecular weight } \\
\text { (kDa); units per vial }\end{array}$ & $\begin{array}{l}\text { Unit equivalents } \\
\text { of ONA }\end{array}$ \\
\hline $\begin{array}{l}\text { OnabotulinumtoxinA } \\
\text { (ONA; Botox }^{\circledast} \text { ) }\end{array}$ & $\begin{array}{l}\text { Medical: axillary } \\
\text { hyperhidrosis, } \\
\text { blepharospasm, } \\
\text { migraine, strabismus. } \\
\\
\text { Cosmetic: glabellar lines, } \\
\text { periocular rhytides }\end{array}$ & A & SNAP25 & $900 ; 100$ & 1 \\
\hline $\begin{array}{l}\text { AbobotulinumtoxinA } \\
\left.\text { (ABO; Dysport }^{\circledast}\right)\end{array}$ & $\begin{array}{l}\text { Medical: blepharospasm, } \\
\text { cervical dystonia. } \\
\text { Cosmetic: glabellar lines. }\end{array}$ & A & SNAP25 & $300-500 ; 500$ & 3 \\
\hline $\begin{array}{l}\text { IncobotulinumtoxinA } \\
\left(\text { INCO; Xeomin }{ }^{\circledast}\right)\end{array}$ & $\begin{array}{l}\text { Medical: blepharospasm, } \\
\text { cervical dystonia. } \\
\text { Cosmetic: glabellar lines. }\end{array}$ & A & SNAP25 & $150 ; 100$ & 1 \\
\hline $\begin{array}{l}\text { PrabotulinumtoxinA- } \\
\text { xvfs (PRA; Jeuveau' }\end{array}$ & Cosmetic: glabellar lines. & A & SNAP25 & $900 ; 100$ & 1 \\
\hline $\begin{array}{l}\text { Rimabotulinumtoxin } \\
\text { (RIMA; Myobloc }{ }^{\circledR} \text { ) }\end{array}$ & $\begin{array}{l}\text { Medical: cervical } \\
\text { dystonia. }\end{array}$ & B & VAMP & $700 ; 5,000$ & N/A \\
\hline
\end{tabular}

N/A: not applicable; ONA: onabotulinumtoxinA; SNAP25: synaptosomal-associated protein 25; VAMP: vesicleassociated membrane protein. 


\section{SAFETY}

The human medial lethal dose of BoNT-A is estimated at $1.3-2.1 \mathrm{mg} / \mathrm{kg}$ (intramuscularly or intravenously), correlating to a median lethal dose (LD50) of 26-42 units/kg; ${ }^{3}$ thus, at the doses used for cosmetic and medical treatment, BoNT is considered safe. Though contraindications to BoNT injection are few, they exist, and include allergy to constituents of BoNT because of the potential for anaphylaxis; neuromuscular disorders such as myasthenia gravis, LambertEaton syndrome, and amyotrophic lateral sclerosis; and pregnancy and nursing. Although medications such as cyclosporine, D-penicillamine, and aminoglycosides are not contraindicated, a thorough medication history is warranted as these drugs may cause neuromuscular junction abnormalities and possibly potentiate the effects of BoNT. ${ }^{4}$

The most common side effects of BoNT administration are pain or sensitivity in the area injected, bruising, swelling, and asymmetry. During injection, it is important that injectors are aware of surrounding musculature as diffusion of BoNT may affect areas that were not intended for treatment. In the literature, the majority of reported adverse events occur after cosmetic treatment of the head and neck for rhytides. Headaches can occur in up to $11.4 \%$ of BoNTA-treated patients, considered to be more likely a result of trauma secondary to injection rather than the BoNT itself, as $20 \%$ of placebo patients also report headache. Headaches can arise up to 2 days post-injection and persist for 2-4 weeks. $^{5}$ When injecting into the platysma, clinicians should assess patients for dysphagia as this is a rare but serious complication, especially when using high doses of BoNT-A. ${ }^{6}$ Generalised reactions to BoNT have been reported in clinical trials, including flu-like symptoms, malaise, nausea, and distant cutaneous reactions such as dermatitis; however, these adverse events are uncommon and self-resolve. ${ }^{7}$

Although immunogenicity to the $150 \mathrm{kDa}$ toxin of BoNT-A was originally reported, it is rare in patients treated after 1997. New formulations of BoNT-A contain only $20 \%$ of the original protein and are hypothesised to decrease the likelihood for patient immune reaction. However, cases of immunogenicity even to newer BoNT-A formulations are occasionally reported; for instance, a patient developed neutralising antibodies after treatment of masseter hypertrophy using Vistabe ${ }^{\circledR}$ (Allergan Inc., Irvine, California, USA) and Azzalure (Galderma S.A., Paris, France). Risks for increased immunogenicity include large BoNT-A doses, frequent injection, and short intertreatment duration ( $<3$ months). ${ }^{7}$

\section{MATERIALS AND METHODS}

A systematic review of the PubMed database using Preferred Reporting Items for Systematic Reviews and Meta-Analyses (PRISMA) guidelines was completed in December 2020 using the search term "botulinum toxin AND (flushing OR erythema OR rosacea OR menopause OR oily OR sebum OR rhytides OR wrinkles OR lines OR mood OR hyperhidrosis OR alopecia OR bullous OR Hailey-Hailey OR pemphigus OR linear IgA OR epidermolysis bullosa OR scar OR keloid OR Raynaud OR hidradenitis suppurativa OR psoriasis)," which resulted in a total of 2,951 total articles. Inclusion criteria were manuscripts written in English pertaining to the use of botulinum toxin in dermatologic conditions in the past 10 years; review papers, commentaries, editorials, practice guidelines, animal studies, and articles not written in English were excluded from review.

\section{RESULTS}

After inclusion and exclusion criteria were applied, 404 studies were reviewed, with 208 discussing cosmetic uses of botulinum toxin such as rhytides or facial rejuvenation $(n=185)$, flushing $(n=13)$, depression or psychologic effects after rhytide treatment $(n=7)$, and oily skin $(n=3)$; while 196 discussed medical uses including hyperhidrosis $(n=100)$, abnormal scarring $(n=32)$, Raynaud's phenomenon ( $n=25)$, bullous disease $(n=10)$, alopecia $(n=5)$, as well as other disease processes $(n=24)$.

\section{Cosmetic Uses}

\section{Flushing}

Flushing or erythema of the scalp, face, neck, and chest can be caused by a variety of underlying neurogenic conditions such as rosacea or 
symptomatic menopause. Although the use of BoNT has mixed results for the treatment of flushing, multiple studies demonstrate that the injection of 10-500 $\mathrm{U}$ of BoNT-A over affected areas in a $1 \mathrm{~cm}$ grid-like pattern results in decreased symptomatology (such as 'hot flashes' or perspiration) and improved patient quality of life as measured by the Dermatology Quality of Life Index (DLQI) over 6 months.

\section{Oily skin}

BoNT-A has been trialled for the treatment of excess sebum production of the forehead and 'T-zone'. Although it is not known how BoNT affects sebaceous glands, it is possible that the toxin targets local muscarinic receptors and arrector pili muscles, thus regulating sebum production. Preliminary retrospective data show that patients treated with BoNT report decreased sebum production and pore size. A more recent prospective study injected 30-45 $U$ of $A B O$ to the foreheads of 25 subjects, with $91 \%$ of patients reporting a decrease in sebum production and a reduction in pore size after assessment of photographs. ${ }^{10}$

\section{Rhytides}

Overactive musculature, photodamage, and ageing cause the formation of dynamic and static rhytides (i.e., 'wrinkles') that patients perceive as making their appearance fatigued or angry; treatment of facial rhytides can lead to a more relaxed and rested appearance. Although currently the FDA has only approved BoNT for treatment of glabellar (corrugator supercilii, depressor supercilii, and procerus muscles) and periorbital lines ('crow's feet'; orbicularis oculi muscle), off-label use of BoNT occurs for the treatment of horizontal forehead lines (frontalis muscle), ptotic brow (lateral orbicularis oculi), horizonal nasal lines ('bunny lines'; nasalis muscle), 'gummy smile' (levator labii superioris alaeque nasi muscle), perioral lines (orbicularis oris muscle), dimpled chin (mentalis muscle), 'downturned smile' (depressor anguli oris), masseter hypertrophy (masseter muscle), mandibular border ('Nefertiti lift'; superior platysmal band), and platysmal bands of the neck (platysma muscle), with clinical results lasting approximately 3 months (Figure 1). 6,11

\section{Combination therapy}

Combining BoNT with filler injection for simultaneous treatment of dynamic rhytides and volume loss is popular in the outpatient setting. Hyaluronic acid and calcium hydroxyapatite fillers in combination with BoNT have been used successfully in multiple areas of the face, including the glabella, periorbital or perioral areas, nasolabial folds, as well as the jaw and chin. Filler should be placed before BoNT to avoid migration of toxin that may occur when injected filler is massaged. It has even been suggested that the combination of filler and BoNT can increase the duration of filler clinical efficacy in the glabellar area to almost double because of decreased facial movement. ${ }^{5}$

BoNT should be avoided on the same day as laser treatment because of concerns for toxin diffusion. Although no studies have shown BoNT diffusion after microfocused/highintensity focused ultrasound or radiofrequency microneedling treatment, it is reasonable to assume that diffusion may occur after such modalities and the same caution should be considered.

\section{Psychological improvement}

In conjunction with rhytide reduction, BoNT improves patient mood and perceived confidence. Improvements in the FACE-Q (satisfaction with facial appearance, satisfaction with facial skin, and appraisal of facial lines) score after treatment of moderate-to-severe glabellar lines have been reported. Even after 120 days, when clinical effects of BoNT should be waning, patients have reported increased psychological well-being and improved facial appearance.

Given the impact of BoNT impact on mood lasting beyond its effect on musculature, clinicians should discuss with patients at which point retreatment is necessary to achieve maximal psychological and clinical response, rather than automatically reinjecting BoNT every 3 months. ${ }^{12,13}$ In addition, BoNTs have been used in neurology for the successful treatment and prevention of migraines, with a notable increase in patient well-being and quality of life. ${ }^{14}$ 


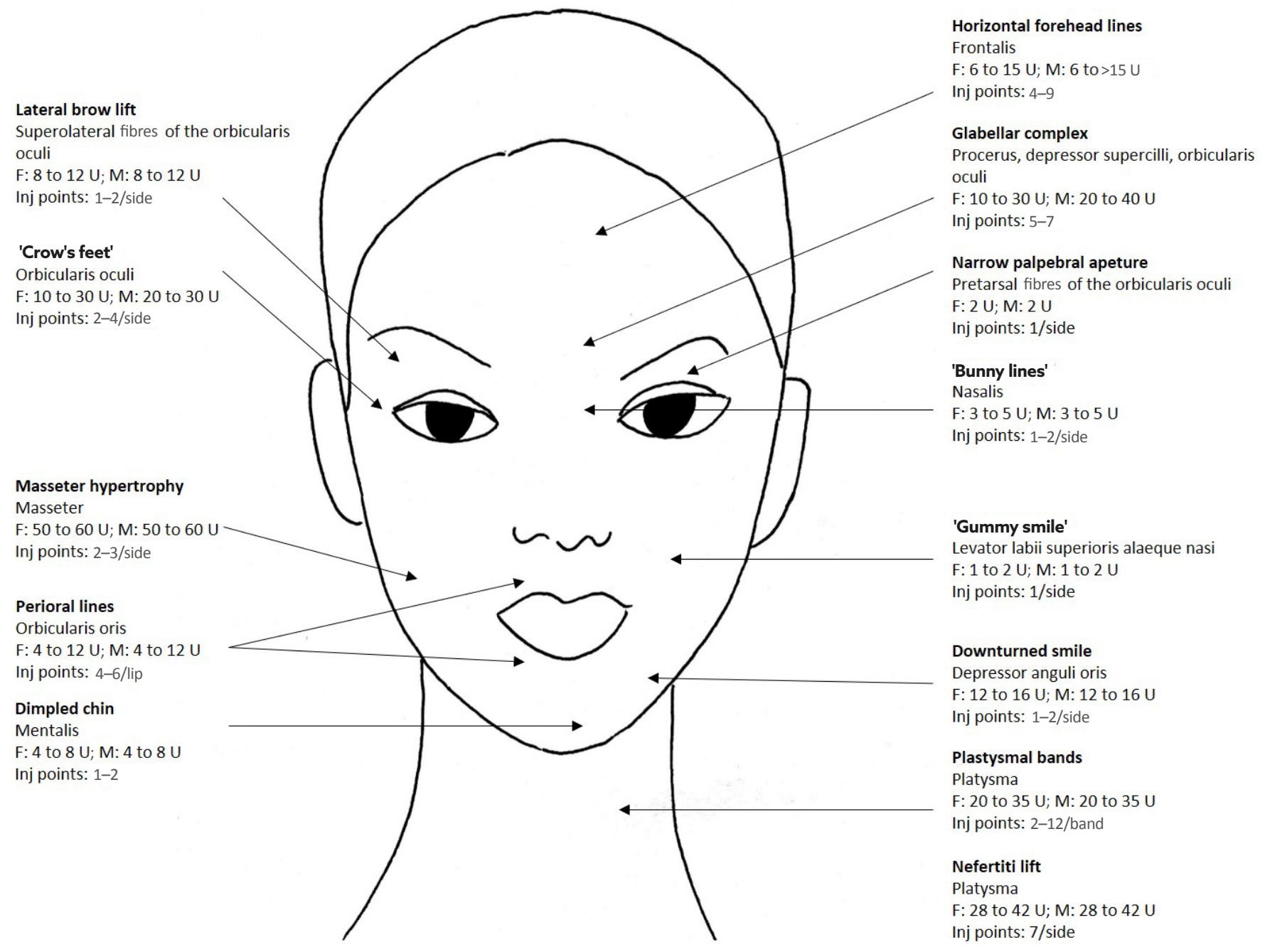

Figure 1: Target areas for BoNT-A treatment of the face.

BoNT-A treatment of rhytides of the upper, mid, and lower face, with suggested total ONA units for female and male patients, as well as number of injection points. ${ }^{32}$

BoNT: botulinum toxin; F: female; inj: injection; M: male; ONA: onabotulinumtoxinA.

Table 2: Protocols for the treatment of primary hyperhidrosis using BoNT with suggested total ONA units, as well as number of injection points. ${ }^{19}$

\begin{tabular}{|l|l|}
\hline Anatomic location of primary hyperhidrosis & Injection protocol \\
\hline Axillae & $\begin{array}{l}50-100 \mathrm{U} / \text { axilla } \\
\text { Inject every 1-2 cm (approximately 15 injection sites) }\end{array}$ \\
\hline Palms & $\begin{array}{l}75-100 \mathrm{U} / \text { palm } \\
\text { Inject every 1 cm, 2-3 injection points per digit } \\
\text { (approximately 35-50 injection sites) }\end{array}$ \\
\hline Soles & $\begin{array}{l}100-200 \mathrm{U} / \mathrm{sole} \\
\text { Inject every 1 cm, 2-3 injection points per digit } \\
\text { (approximately 35-50 injection sites) }\end{array}$ \\
\hline
\end{tabular}

BoNT: botulinum toxin; ONA: onabotulinumtoxinA. 


\section{Medical Uses}

Within the purview of dermatologic practice, the only FDA-approved medical use of BoNT is ONA for the treatment of axillary hyperhidrosis. Despite further lack of approval, BoNT has been used off-label for the treatment of alopecia, bullous skin disorders, palmar and plantar hyperhidrosis, hypertrophic and keloidal scarring, as well as other dermatologic medical conditions such as Raynaud's phenomenon, hidradenitis suppurativa, and psoriasis.

\section{Hyperhidrosis}

BoNT disrupts acetylcholine-induced secretion from eccrine sweat glands, which are located in the axillae, as well as throughout the body. ONA is approved by the FDA for the treatment of primary axillary hyperhidrosis. The use of BoNT for treatment of primary hyperhidrosis has extended to use in the palms and soles, as well as other areas of the body. Adolescent patients as young as 12 years old have been treated successfully with BoNT, with some researchers advocating for early treatment of hyperhidrosis to decrease future patient morbidity such as decreased quality of life. ${ }^{15}$

Treatment of the primary axillary hyperhidrosis can be accomplished using 50-100 $U$ of BoNT-A per axilla with intradermal injections spaced every 1-2 cm in a grid-like pattern (for approximately 15 injection sites). Clinical results are noticeable after 1 week and last for 3-10 months. Patients are generally satisfied with treatment. It is important to inform patients that compensatory sweating can occur in up to $5 \%$ of cases post-injection., 46,17 Treatment of palmar and plantar hyperhidrosis can also be accomplished with BoNT. Injections should be spaced $1 \mathrm{~cm}$ apart in a grid-like pattern with 2-3 injection points per digit (for approximately 35-50 injection sites). Each hand can receive 75-100 $U$ of BoNT-A, while each foot can receive 100-200 $\mathrm{U}$. Clinical results take up to 1 week for effect and last 3-6 months. Specific adverse events associated with palmar and plantar injection of BoNT should be discussed with patients prior to treatment. After palmar injection, patients can experience weakness, especially in pinch strength, while plantar injections may impede walking, especially if a nerve block is performed prior to BoNT treatment. ${ }^{18,19}$ Unfortunately, 20\% of patients injected with BoNT for plantar hyperhidrosis will not respond to treatment (Table 2). ${ }^{19}$

Due to the painful nature of injections into the palms and soles, discomfort during BoNT injections has been mitigated in a variety of ways. Studies have demonstrated that topical lidocaine/prilocaine, vibration, cryoanalgesia, repeated needle replacement, use of microneedles, intravenous administration of anaesthesia and peripheral nerve blocks, iontophoretic administration of $2 \%$ lidocaine, needle-free units to administer local anaesthesia using pressure, and inhaled nitrous oxide are all viable methods of analgesia during injection. ${ }^{17,19-25}$ Advances in BoNT delivery may also decrease injectionassociated pain. Needle-free jet injection devices have been trialled for the treatment of palmar hyperhidrosis (Med-Jet BMX, Medical International Technologies, Montréal, Canada); however, clinical efficacy was decreased as compared to traditional injections. ${ }^{26} \mathrm{An}$ in vivo murine study demonstrated that BoNTA-coated polylactic acid microneedles are a safe and effective delivery method, resulting in significantly decreased sweating. ${ }^{27}$ Delivery of BoNT-A using a combined fractional ablative laser and ultrasound has been trialled successfully to treat palmar hyperhidrosis. ${ }^{28} \mathrm{~A}$ topical formulation of BoNT-A in a liposomal base applied nightly for 7 nights was found to be effective at reducing axillary sweat for up to 8 weeks, compared to vehicle alone, suggesting that painless delivery methods for BoNT are on the horizon. ${ }^{29}$

Recent studies have employed BoNT to treat hyperhidrosis in novel locations. For example, one case report injected $100 \mathrm{U}$ of BoNT-A every 6-8 months at the gluteal cleft of a male patient with pressure-induced ulceration to decrease sweat production and associated wound maceration; over a period of 2 years, skin integrity was maintained with no clinical worsening of the pressure injury. ${ }^{30}$ Another study employed a total of $2250 \mathrm{U}$ of BoNT-B (Neurobloc, Eisai Europe, Hatfield, UK) injected every $15 \mathrm{~mm}$ across the forehead, frontal scalp, parietal scalp, and occipital scalp in a band-like pattern, as well as periocular and perioral, for the treatment of postmenopausal craniofacial hyperhidrosis. Three weeks after 
treatment, patients treated with BoNT-B reported a $91 \%$ improvement in the DLQI, compared with an $18 \%$ decrease in quality of life with placebo injections. ${ }^{31}$ In addition, BoNT injection has been shown to be clinically effective for the therapy of Frey's syndrome and sialorrhoea; however given the anatomic location of injections, treatment is often performed by otolaryngology. ${ }^{32,33}$

In addition to hyperhidrosis, BoNT-A has been used successfully to treat chromhidrosis (coloured sweat of the axillae and cheek) and bromhidrosis (malodorous sweat of the axillae and genitals). Treatment protocols generally follow the same recommendations as for axillary hyperhidrosis, with patients receiving approximately $100 \mathrm{U}$, divided across the affected area(s), per treatment session. Clinical results last from 3 to 9 months. ${ }^{34,35}$ Furthermore, small studies demonstrate improvement in hand and dyshidrotic eczema (pompholyx) in patients with palmar hyperhidrosis after injection of $100 \mathrm{U}$ of BoNT-A. ${ }^{36}$

\section{Alopecia}

BoNT-A has been used for the treatment of androgenetic alopecia, cephalalgic alopecia, alopecia areata, and radiation-induced alopecia. Although it is unknown how BoNT contributes to hair regrowth, it is hypothesised that decreasing microvascular pressure through muscle relaxation may increase oxygen delivery to the hair follicles. Treatment protocols range from 30 to $150 \mathrm{U}$ injected into the frontal, temporal, periauricular, and occipital musculature over 1-12 sessions. Most studies report clinical improvement in hair growth or density and high levels of patient satisfaction; however, further randomised controlled trials are necessary to determine the true effect of BoNT on hair growth. ${ }^{37-39}$ On the contrary, multiple BoNT injections for forehead wrinkles have been associated with the development of frontal alopecia. ${ }^{40}$

\section{Bullous skin disease}

BoNT has been trialled off-label for the treatment of several bullous skin disorders including Hailey-Hailey disease (familial benign pemphigus), linear IgA bullous dermatosis, and localised epidermolysis bullosa simplex (Weber-Cockayne). Hailey-Hailey

disease has been treated with BoNT-A injections, as well as a combination of BoNT-A and erbium: yttrium aluminum garnet ablative laser or oral tacrolimus, in the axillae, inframammary, groin and intergluteal cleft regions. Doses range from 25 to $200 \mathrm{U}$ every 3 to 6 months with clinical improvement lasting at least 12 months after treatment. ${ }^{41,42}$ Case reports have reported the treatment of the bilateral axillae in a young patient with linear IgA bullous dermatosis using $50 \mathrm{U}$ per axilla, and $100 \mathrm{U}$ injected into the foot of a middle-aged female affected by localised epidermolysis bullosa simplex.43,44

\section{Raynaud's phenomenon}

Raynaud's phenomenon, vasospasm of the digits, is difficult to treat and is often recalcitrant to first-line therapies such as calcium channel blockers, nitrates, phosphodiesterase inhibitors, iloprost, and bosentan. Surgical procedures such as sympathectomy are invasive and require recovery and downtime. BoNT injection has been used successfully for the treatment of primary and sclerosis-associated Raynaud's phenomenon of the fingers. ${ }^{45,46}$ After injection of 50-100 $U$ of BoNT-A in 19 patients with Raynaud's phenomenon, investigators noted that 13 patients reported immediate improvement in pain and chronic ulcerations healed within 60 days. ${ }^{47}$ When compared to normal saline injections, digital pulp temperature significantly improved in the fingertips treated with BoNT after 6 weeks, suggesting BoNT is effective at treating Raynaud's phenomenonassociated vasospasm. ${ }^{48}$ Currently, there are no standardised injection protocols used for treatment; one study demonstrated that injection at the wrist, digits, or distal metacarpus does not result in significantly different clinical outcomes, although all were effective at treating Raynaud's phenomenonassociated vasospasm. ${ }^{49}$

\section{Scarring}

BoNT has been used to decrease hypertrophic and keloidal scarring resulting from increased wound edge tension; by paralysing the surrounding musculature, tensile forces and abnormal scar formation may be decreased. It has also been suggested that BoNT may decrease TGF- $\beta$ signalling, a regulator of hypertrophic scarring. ${ }^{50}$ Studies demonstrated 
the use of 15-140 $U$ of BoNT-A administered up to 9 days post-procedure, every 4-12 weeks for 1-3 treatment sessions resulted in scar softening, reduced elevation, and decreased symptoms such as itching and pain. ${ }^{51}$ However, one study using a three-dimensional optical system to image keloidal scars, showed no keloidal tissue regression after treatment with BoNT. ${ }^{52}$ When compared to intralesional triamcinolone $10 \mathrm{mg} / \mathrm{cc}$ every 4 weeks for 6 injection sessions, $5 \mathrm{U} / \mathrm{cm}^{3}$ of BoNT-A every 8 weeks for 3 sessions was found to significantly improve subjective complaints such as itch, pain, and allodynia, possibly due to the clinical effects of BoNT-A on small-fibre neuropathy; however, only intralesional triamcinolone resulted in significant improvement in keloid scar softening..$^{53}$

Combination therapy using BoNT-A has also been used for the treatment of hypertrophic or keloidal scarring. Clinical improvement of a traumatic scar of the chin was achieved with injection of 6-8 $U$ of BoNT-A in combination with 4 treatments of $595 \mathrm{~nm}$ pulsed dye laser every 2 weeks. ${ }^{54}$ A combination of INCO and microneedling of facial scars after non-melanoma skin cancer surgery results in significantly improved cosmetic results and patient satisfaction with appearance post-surgery. ${ }^{55}$

\section{Other medical diseases}

Case reports have reported use of 40-250 U of BoNT-A per site (axilla, inguinal folds) with 1-3 treatment sessions for hidradenitis suppurativa, with complete remission of disease lasting 6 months to 3 years. Like bullous diseases, it is thought that decreased sweat production contributes to hidradenitis suppurativa improvement by eliminating an environment in which bacteria may flourish, and preventing rupture of the follicular unit. ${ }^{56,57}$ BoNT-A has also been trialled as an adjuvant for the treatment of aquagenic keratoderma, ${ }^{58}$ congenital eccrine naevus, ${ }^{53}$ Darier Disease (keratosis follicularis), ${ }^{59}$ inverse and plaque psoriasis, ${ }^{60}$ notalgia paraesthetica, ${ }^{61}$ pachyonychia congenita, ${ }^{62}$ and post-herpetic neuralgia resulting from herpes zoster. ${ }^{63}$

\section{THE FUTURE}

Multiple new formulations of BoNT-A are currently being trialled for the treatment of glabellar and periocular lines. DaxibotulinumtoxinA (DAXI; Revance Therapeutics Inc., Newark, New Jersey, USA) has been studied as both a topical and injectable therapy; but, the topical preparation was not efficacious. Injectable DAXI has entered Phase III FDA trials, with efficacy treating glabellar lines and clinical results possibly lasting 5 weeks longer than ONA (SAKURA 1 and 2, BELMONT). ${ }^{64}$ LetibotulinumtoxinA (LETI; Botulax ${ }^{\circledast}$, Hugel, Chuncheon, South Korea) is currently commercially available in Asia and is undergoing studies in anticipation of FDA approval for treatment of periorbital rhytides. ${ }^{65}$ When compared to INCO, LETI has a greater amount of neurotoxin protein per volume, but also has increased amounts of inactive neurotoxin, which hypothetically may increase the risk of immunoreaction. ${ }^{66}$

Liquid formulations of BoNT-A are being studied to reduce current concerns for reconstitution contamination or error. NivobotulinumtoxinA (NIVO; Innotox ${ }^{\circledast}$, Medytox, Inc., Seoul, South Korea, and MT10109L, Allergan, Inc., Irvine, California, USA) is non-inferior to ONA for the treatment of frown lines, and is undergoing studies to determine clinical efficacy for glabellar lines. ${ }^{67,68}$ QM-1114 (Galderma Laboratories, L.P., Lausanne, Switzerland) is superior for the treatment of glabellar lines compared to placebo, and is currently being studied for the reduction of lateral canthal lines. ${ }^{69-71}$

In addition to new formulations of BoNT-A, liquid BoNT-E (ED-001, Bonti, Inc., Newport Beach, California, USA) is being pursued due to its purported faster onset of action (24 hours post-injection) and decreased duration of clinical results (14-30 days). EB001 has been shown to safely and effectively decrease appearance of glabellar lines and may improve forehead scar cosmesis post-Mohs micrographic surgery (SHINE). ${ }^{72,73}$ It is possible that in addition to the current cosmetic indications being pursued by pharmaceutical companies, dermatologists will be able to 
use these new BoNT-A formulations for the off-label treatment of dermatologic medical disease as outlined above.

\section{CONCLUSION}

BoNT is an extremely versatile injectable medication that can be used for cosmetic, as well as medical, treatment of dermatologic diseases such as hyperhidrosis, hair loss, aberrant scarring, bullous skin disorders, psoriasis, and Raynaud's phenomenon, amongst others. Although the use of BoNT use is relatively safe, it is always important to be aware of injection points as the toxin may diffuse and negatively affect adjacent areas that should not have been treated. Clinicians should be acutely aware of site-specific adverse events, especially with injection of BoNT into the neck, hands, or feet. Dermatologists need to be educated on both the on- and off-label uses of BoNT to provide patients with appropriate treatment and decrease associated morbidity. Well-developed clinical trials are required to determine the true clinical efficacy of BoNT in the off-label setting, as well as possible longterm safety concerns.

\section{References}

1. Carruthers JD, Carruthers JA. Treatment of glabellar frown lines with C. botulinum-A exotoxin. J Dermatol Surg Oncol. 1992;18:17-21.

2. Huang $W$ et al. Pharmacology of botulinum toxin. J Am Acad Dermatol. 2000;43:249-59.

3. Scott $A B$, Suzuki D. Systemic toxicity of botulinum toxin by intramuscular injection in the monkey. Mov Disord. 1988;3:333-5

4. Coté TR et al. Botulinum toxin type A injections: adverse events reported to the US Food and Drug Administration in therapeutic and cosmetic cases. J Am Acad Dermatol. 2005;53:407-15

5. Carruthers J, Carruthers A. A prospective, randomized, parallel group study analyzing the effect of BTX-A (Botox) and nonanimal sourced hyaluronic acid (NASHA, Restylane) in combination compared with NASHA (Restylane) alone in severe glabellar rhytides in adult female subjects: treatment of severe glabellar rhytides with a hyaluronic acid derivative compared with the derivative and BTX-A. Dermatol Surg. 2003;29:802-9.

6. Carruthers J, Carruthers A. Practical cosmetic botox techniques. J Cutan Med Surg. 1999;3(4):S49-52.

7. Naumann M et al. Meta-analysis of neutralizing antibody conversion with onabotulinumtoxinA (BOTOX ${ }^{\circledR}$ ) across multiple indications. J Mov Disord. 2010;25: 2211-8

8. Odo MEY et al. Botulinum toxin for the treatment of menopausal hot flushes: a pilot study. Dermatol Surg. 2011;37:1579-83.

9. Park $\mathrm{KY}$ et al. Botulinum toxin for the treatment of refractory erythema and flushing of rosacea. Dermatology. 2015;230:299-301.

10. Rose AE, Goldberg DJ. Safety and efficacy of intradermal injection of botulinum toxin for the treatment of oily skin. Dermatol Surg. 2013;39:4438.

11. Jensen J et al, "Botulinum toxins," Draelos ZD (eds.), Cosmetic dermatology: products and procedures (2015) 2nd edition, Chichester: John Wiley \& Sons, Ltd, pp.364-74.

12. Chang $B L$ et al. Patient perceived benefit in facial aesthetic procedures: FACE-Q as a tool to study botulinum toxin injection outcomes. Aesthet Surg J. 2016;36:810-20.

13. Cohen $\mathrm{J}$ et al. Assessment of psychological well-being after abobotulinumtoxinA treatment: a comparison of 2 reconstitution volumes. Dermatol Surg.2020;46:289 92.

14. Burstein R et al. Mechanism of action of onabotulinumtoxinA in chronic migraine: a narrative review. Headache. 2020;60(7):1259-72.

15. Bernhard M et al. Sweaty feet in adolescents-early use of botulinum type A toxin in juvenile plantar hyperhidrosis. Pediatr Dermatol. 2018;35:784-6

16. Doft $M$ et al. Treatment of axillary hyperhidrosis with botulinum toxin: a single surgeon's experience with 53 consecutive patients. Aesthetic Plast Surg. 2011;35:1079-86.

17. Carruthers JD, Carruthers JA, "Focal axillary hyperhidrosis," Glaser D, Mattox AR (eds.), Botulinum toxin: procedures in cosmetic dermatology (2018) 4th edition, Toronto: Elsevier Inc, pp.177-85.

18. Klein AW. Contraindications and complications with the use of botulinum toxin. Clin Dermatol. 2004;22:66-75.

19. Carruthers JD, Carruthers JA,
"Palmoplantar hyperhidrosis," Mariwalla K, Solish N (eds.), Botulinum toxin: procedures in cosmetic dermatology (2018) 4th edition, Toronto: Elsevier Inc, pp.18792.

20. Benohanian A. Needle-free anaesthesia prior to botulinum toxin type A injection treatment of palmar and plantar hyperhidrosis. $\mathrm{Br} \mathrm{J}$ Dermatol. 2007;156:593-6.

21. Richards RN. Ethyl chloride spray for sensory relief for botulinum toxin injections of the hands and feet. J Cutan Med Surg. 2009;13:253-6.

22. Torrisi BM et al. Pocketed microneedles for rapid delivery of a liquid-state botulinum toxin A formulation into human skin. J Control Release. 2013;165:146-52.

23. Blaheta $\mathrm{H}$ et al. Intravenous regional anesthesia (Bier's block) is superior to a peripheral nerve block for painless treatment of plantar hyperhidrosis with botulinum toxin. J Am Acad Dermatol. 2003;48:302-4.

24. Campanati A et al. Local neural block at the wrist for treatment of palmar hyperhidrosis with botulinum toxin: technical improvements. J Am Acad Dermatol. 2004;51:345-8.

25. Reed ML. Surgical pearl: mechanoanesthesia to reduce the pain of local injections. J Am Acad Dermatol. 2001;44:671-2.

26. Vadeboncoeur $S$ et al. Treatment of palmar hyperhidrosis with needle injection versus lowpressure needle-free jet injection of onabotulinumtoxinA: an open-label prospective study. Dermatol Surg. 2017;43:264-9.

27. Shim DH et al. Development of botulinum toxin A-coated microneedles for treating palmar hyperhidrosis. Mol Pharm. 2019;16:4913-9. 
28. Issa $\mathrm{M}$ et al. Early investigations in drug delivery of onabotulinum toxin A using combined fractional ablative laser with impact ultrasound vs. injections of onabotulinum toxin A for palmar hyperhidrosis: a rightleft comparison trial. Br J Dermatol. 2018;179:1168-9.

29. Lueangarun $\mathrm{S}$ et al. Topical botulinum toxin type A liposomal cream for primary axillary hyperhidrosis: a double-blind, randomized, split-site, vehicle-controlled study. Dermatol Surg. 2018;44:1094-101.

30. Uhlig-Reche et al. Treatment of secondary hyperhidrosis of the intergluteal cleft with botulinum toxin. PM R. 2020;DOI:10.1002/ pmrj.12404.

31. Cabreus P et al. Postmenopausal craniofacial hyperhidrosis treated with botulinum toxin type B. J Dermatol. 2019;46(10):874-8.

32. Freni $F$ et al. Use of botulinum toxin in Frey's syndrome. Clin Case Rep. 2019;7:482-5.

33. Weitzman RE et al. A 10-year retrospective review of botulinum toxin injections and surgical management of sialorrhea. Cureus. 2020;12:e7916.

34. Beer K, Oakley H. Axillary chromhidrosis: report of a case, review of the literature and treatment considerations. J Cosmet Dermatol. 2010;9:318-20.

35. Wu CJ et al. Efficacy and safety of botulinum toxin a in axillary bromhidrosis and associated histological changes in sweat glands: a prospective randomized doubleblind side-by-side comparison clinical study. Dermatol Surg. 2019;45:1605-9.

36. Swartling $C$ et al. Treatment of dyshidrotic hand dermatitis with intradermal botulinum toxin. J Am Acad Dermatol. 2002;47:667-71.

37. Cho HR. Treatment effects of intradermal botulinum toxin type A injection on alopecia areata. Dermatol Surg. 2010;36(Suppl 4):2175-81.

38. Hyun $M$ et al. Radiation-induced alopecia treated with botulinum toxin type A injection. Plast Reconstr Surg Glob Open. 2014;2:e226.

39. Shon $U$ et al. The effect of intradermal botulinum toxin on androgenetic alopecia and its possible mechanism. J Am Acad Dermatol. 2020;83(6):1838-9.

40. Di Pietro A, Piraccini BM. Frontal alopecia after repeated botulinum toxin type A injections for forehead wrinkles: an underestimated entity? Skin Appendage Disord. 2016;2:67-9.

41. Bedi M, Taylor AL. Recalcitrant Hailey-Hailey disease responds to ora tacrolimus and botulinum toxin type A. Cutis. 2015:96:e14-16.

42. Ho D, Jagdeo J. Successful botulinum toxin (onabotulinumtoxinA) treatment of Hailey-Hailey disease. Drugs Dermatol. 2015;14:68-70.

43. Abitbol RJ, Zhou LH. Treatment of epidermolysis bullosa simplex, Weber-Cockayne type, with botulinum toxin type A. Arch Dermatol. 2009;145:13-5.

44. Legendre $L$ et al. Botulinum toxin A: an effective treatment for linear immunoglobulin A bullous dermatosis located in the axillae. Acta Derm Venereol. 2016:96:122-3.

45. Herrick AL. The pathogenesis, diagnosis and treatment of Raynaud phenomenon. Nat Rev Rheumatol. 2012;8:469-79

46. Smith $L$ et al. Botulinum toxin-A for the treatment of Raynaud syndrome. Arch Dermatol. 2012;148:426-8.

47. Neumeister MW et al. Botox therapy for ischemic digits. Plast Reconstr Surg. 2009;124:191-201.

48. Jenkins $\mathrm{SN}$ et al. A pilot study evaluating the efficacy of botulinum toxin $A$ in the treatment of Raynaud phenomenon. J Am Acad Dermatol. 2013:69:834-5.

49. Fregene et al. Botulinum toxin type A: a treatment option for digital ischemia in patients with Raynaud's phenomenon. J Hand Surg. 2009;34:446-52.

50. Elhefnawy AM. Assessment of intralesional injection of botulinum toxin type A injection for hypertrophic scars. Indian J Dermatol Venereol Leprol. 2016;82:279-83.

51. Xiao Z et al. Treatment of hypertrophic scars with intralesional botulinum toxin type A injections: a preliminary report. Aesthetic Plast Surg. 2009;33:409-12.

52. Gauglitz GG et al. Botulinum toxin A for the treatment of keloids. Skin Pharmacol Physiol. 2012;25:313-8.

53. Shaarawy E et al. Intralesional botulinum toxin type A equally effective and better tolerated than intralesional steroid in the treatment of keloids: a randomized controlled trial. J Cosmet Dermatol. 2015;14:1616.

54. Lee SJ et al. Combined treatment with botulinum toxin and 595 $\mathrm{nm}$ pulsed dye laser for traumatic scarring. Ann Dermatol. 2015;27:7568.

55. Casabona GR, Giacomo TB. Improving the appearance of surgica facial scars with incobotulinumtoxinA and microneedling. J Drugs Dermatol. 2020;19:611-5.

56. Khoo ABS, Burova EP. Hidradenitis suppurativa treated with Clostridium botulinum toxin A. Clin Exp Dermatol. 2014;39:749-50.

57. Martina $\mathrm{E}$ et al. Botulinum toxin in dermatology A review- hidradenitis suppurativa: how to treat with
BoNT-A. Skin Appendage Disord. 2017;3(1):39-56.

58. Bagazgoitia L et al. Letter: aquagenic keratoderma: successful treatment with botulinum toxin. Dermatol Surg 2010:36;434-6.

59. Santiago-et-Sánchez-Mateos JL et al. Botulinum toxin type $A$ for the preventive treatment of intertrigo in a patient with Darier's disease and inguinal hyperhidrosis. Dermatol Surg 2008;34:1733-7.

60. Gilbert E, Ward NL. Efficacy of botulinum neurotoxin type $A$ for treating recalcitrant plaque psoriasis. J Drugs Dermatol. 2014;13:1407-8.

61. Maari $\mathrm{C}$ et al. Treatment of notalgia paresthetica with botulinum toxin A: a double-blind randomized controlled trial. J Am Acad Dermatol. 2014;70:1139-41.

62. González-Ramos J et al. Efficacy of botulinum toxin in pachyonychia congenita type 1: report of two new cases. Dermatol Ther. 2016;29:32-6.

63. Apalla $Z$ et al. Botulinum toxin $A$ in postherpetic neuralgia: a parallel, randomized, double-blind, singledose, placebo-controlled trial. Clin J Pain. 2013:29:857-64.

64. Bertucci $\vee$ et al. DaxibotulinumtoxinA for Injection has a prolonged duration of response in the treatment of glabellar lines: pooled data from two multicenter, randomized, doubleblind, placebo-controlled, phase 3 studies (SAKURA 1 and SAKURA 2). J Am Acad Dermatol. 2020;82:838-45.

65. Hugel, Inc. Evaluate the Safety and Efficacy of Botulax ${ }^{\oplus}$ as Compared to Botox ${ }^{\circledast}$ in Subject With Moderate to Severe Crow's Feet Lines. NCT03408236. https://clinicaltrials. gov/ct2/show/NCT03408236.

66. Frevert $\mathrm{J}$ et al. Comparison of botulinum neurotoxin type $A$ formulations in Asia. Clin Cosmet Investig Dermatol. 2018;11:327-31.

67. Allergan, Inc. MT10109L in the Treatment of Glabellar Lines. NCT03795922. https://clinicaltrials. gov/ct2/show/NCT03795922.

68. Kim J E et al. The efficacy and safety of liquid-type botulinum toxin type A for the management of moderate to severe glabellar frown lines. Plast Reconstr Surg. 2015;135:732-41.

69. Q-Med AB. Long-term Treatment of Moderate to Severe Glabellar Lines and Lateral Canthal Lines (READY-4). NCT04225260. https://clinicaltrials. gov/ct2/show/NCT04225260.

70. Q-Med AB. Treatment of Moderate to Severe Lateral Canthal Lines (READY-2). NCTO4249687. https:// clinicaltrials.gov/ct2/show/ NCT04249687

71. Monheit $\mathrm{G}$ et al. Evaluation of QM-1114, a novel ready-touse liquid botulinum toxin, in 
aesthetic treatment of glabellar lines. 2019. Available at: https:// www.wcd2019milan-dl.org/ abstract-book/documents/latebreaking-abstracts/O3-aestheticcosmetic-dermatology/evaluationof-qm1114-a-novel-490.pdf. Last accessed: April 2021.
72. Yoelin SG et al. Safety and efficacy of EB-001, a novel type E botulinum toxin, in subjects with glabellar frown lines: results of a phase 2 , randomized, placebo-controlled, ascending-dose study. Plast Reconstr Surg. 2018;142:847e-55e.
73. Rodrigues O; Bonti. Bonti Announces Successful Completion of Phase 2a Scar Reduction Clinical Study. 2018. Available at: https://www.

biospace.com/article/releases/bontiannounces-successful-completionof-phase-2a-scar-reduction-clinicalstudy/. Last accessed : April 2021. 